\title{
Effects of Tai Chi Yunshou exercise on community-based stroke patients: a cluster randomized controlled trial
}

Guanli Xie ${ }^{1,3 \dagger}$, Ting Rao ${ }^{2 \dagger}$, Lili Lin ${ }^{1}$, Zhengkun Lin ${ }^{1}$, Tianshen Xiao ${ }^{1,4}$, Ming'ge Yang ${ }^{1,4}$, Ying Xu ${ }^{1,3}$, Jinmei Fan ${ }^{1}$, Shufang $\mathrm{Lin}^{2}$, Jinsong $\mathrm{Wu}^{1,5}$, Xiaodong Feng ${ }^{6}, \mathrm{Li} \mathrm{Li}^{7}$, Jing TaO ${ }^{1,4,5^{*}}$ and Lidian Chen ${ }^{1,3,4^{*}}$

\begin{abstract}
Backgroud: Tai Chi Chuan was used for stroke survivors with balance impairments. However, even a short-form of Tai Chi Chuan includes forms that make the exercise challenging for the stroke survivors. Tai Chi Yunshou (wave hands in the cloud) is the "mother" form and the fundamental form of all Tai Chi Chuan styles, which is considered more suitable and feasible for stroke survivors with balance impairments. So this study was designed to evaluate the effects of Tai Chi Yunshou exercise on community-based stroke patients with balance dysfunctions.

Methods: A total of 250 participants from 10 community health centers (5 per arm) were selected and randomly allocated into Tai Chi Yunshou exercise group (TC group) or a balance rehabilitation training group (control group) in an equal ratio. Participants in the TC group were received Tai Chi Yunshou exercise training five times per week for 12 weeks and those in control group were received balance rehabilitation training five times per week for 12 weeks. Outcome assessments including Berg Balance Scale (BBS), Time up to go test (TUGT), Modified Barthel Index (MBI) were measured at baseline, 4 weeks, 8 weeks, 12 weeks and followed-up 6 weeks (18 weeks), 12 weeks (24 weeks). Intention-to-treat analysis was performed. Analysis of variance of repeated measures was used to assess between-group differences.
\end{abstract}

Results: A total of 244 participants, 120 in the TC group and 124 in the rehabilitation group, were included in final analysis. There was no siginificant difference in Tai Chi Yunshou and balance rehabilitation training on the improvement of balance ability and mobility ( $P=0.531$ and $P=0.839$, respectively) after adjustment for baseline. However, there was significant difference between two groups on improvement of motor funtion $(P=0.022)$, fear of falling $(P<0.001)$ and depression $(P=0.035)$ for the post stroke patients. No adverse events were reported during the study.

Conclusion: Tai Chi Yunshou and balance rehabilitation training led to improved balance ability and functional mobility, and both are suitable community-based programs that may benefit for stroke recovery and community reintegration. Our data demonstrated that a 12-week Tai Chi Yunshou intervention was more effective in motor function, fear of falling and depression than balance rehabilitation training. Future studies examining the effectiveness of Tai Chi Yunahou as a balance ability improvement strategy for community-dwelling survivors of stroke are recommended.

Trial registration: Chinese Clinical Trail Registry: ChiCRT-TRC-13003641. Registration date: 22 August, 2013.

Keywords: Tai chi Yunshou exercises, Wave hands in the cloud, Balance dysfunction, Stroke, Community-dwelling, Cluster randomized controlled trial

\footnotetext{
* Correspondence: taojing01@163.com; cld@fjtcm.edu.cn

${ }^{\dagger}$ Guanli Xie and Ting Rao contributed equally to this work.

${ }^{1}$ College of Rehabilitation Medicine, Fujian University of Traditional Chinese

Medicine, 1 Huatuo Road, Minhou Shangjie, Fuzhou 350122, Fujian, China

Full list of author information is available at the end of the article
}

(c) The Author(s). 2018 Open Access This article is distributed under the terms of the Creative Commons Attribution 4.0 International License (http://creativecommons.org/licenses/by/4.0/), which permits unrestricted use, distribution, and reproduction in any medium, provided you give appropriate credit to the original author(s) and the source, provide a link to the Creative Commons license, and indicate if changes were made. The Creative Commons Public Domain Dedication waiver (http://creativecommons.org/publicdomain/zero/1.0/) applies to the data made available in this article, unless otherwise stated. 


\section{Background}

Stroke has become the second most common cause of death and the third most common cause of disability worldwide [1]. Stroke alters people's lives by affecting their motor function, mobility, cognitive function and more, leading to restrictions in their basic activities of daily living (ADL) [2]. Of all the dysfunctions caused by stroke, balance impairment is a common cause of disability and has the greatest impact on ADL performance [3-5], which directly affects the quality of life for patients and their outcomes [6]. Therefore, interventions that could alleviate balance impairments in this target population were suggested to improve their balance and functional mobility [7].

Although many balance programs including standing balance practices [8], and motor relearning programs [9] have been used for the acute and subacute phases of stroke patients, community-dwelling stroke survivors have fewer options due to real and perceived barriers [10]. Moreover, one-on-one sessions are needed for chronic stroke patients [11], which are not easy to obtain in community health centers (CHCs), and supervision by health care professionals is required. These factors all increase the expenditure related to stroke rehabilitation, which becomes a main economic burden for families [12]. So a lower-cost, effective, easily prescribed intervention for stroke survivors with balance dysfunctions is needed in the community [13].

A growing body of evidence has demonstrated that Tai Chi Chuan exercise can help to improve or maintain balance ability, motor function, mobility, fear of falling and quality of life in the elderly $[14,15]$. The safety of Tai Chi Chuan has also been demonstrated among chronic patients including stroke survivors [16-21]. As a mind-body exercise, Tai Chi Chuan can induce relaxation and tranquility of the mind, improve balance function and reduce the risk of falling by enhancing the proprioceptive sensibility which was impaired in stroke survivors [22-25]. In addition, once they have mastered the Tai Chi Chuan forms, stroke survivors can continue to exercise Tai Chi Chuan anywhere, anytime without limitation to guidance or supervision of a profession. Increasing the ability of stroke survivors to perform their own rehabilitation regime anywhere, anytime should also be an important goal for rehabilitation [17]. Tai Chi Chuan was recommend to use in community for stroke patients $[16,17,26]$.

There are multitudinous styles of Tai Chi Chuan, such as Yang-style, Sun-style, and Chen-style, each having short and long forms (form refers to number of movements). The movements vary from style to style. In the current researches, the Yang-style (24-form) [16, 18], Sun-style (12-form) [17], Simplified Tai Chi Chuan (6-form) [27] were used to explore the effects of Tai Chi
Chuan on the balance of stroke survivors. The results indicated that Tai Chi Chuan is a safe, community-based exercise program $[16,26]$, and can improve the balance [28], reduce the fall rate, improve the quality of life $[18,28]$ for this target population. However, even a short-form of Tai Chi Chuan (such as 6-form) included forms (e.g., part the wild horse's mane on both sides, lower the body and stand on one leg) that make the exercise challenging for the stroke survivors, especially those with balance dysfunctions $[10,29]$. Tai Chi Yunshou movement (wave hands in the cloud), known as the "mother form" and the fundamental form of all styles of Tai Chi Chuan, fully embodies the basic pricinple of Tai Chi Chuan [30]. During movement, shoulders are balanced and relaxed, and body weight is evenly distributed on the soles of the feet, the body remains in an upright position with the shoulders aligned over the hips, the waist is the center or axis and directs the movement of the arms, legs, and eyes, the head, trunk, and pelvis rotate as a single unit, aligned over a stable base in the feet [30]. We believe that Tai Chi Yunshou movement maybe suitable and feasible for stroke survivors with balance impairments. The hypothesis that Tai Chi Yunshou movement can improve the balance dysfunction for the community-based stroke survivors was also considered. Thus, a cluster randomized controlled study was designed to evaluate the multi-dimensional effects of a 12-week Tai Chi Yunshou exercise program on physical health and mental health for a group of community-dwelling stroke survivors.

\section{Methods \\ Participants}

A single-blind cluster randomized, parallel-controlled trial was conducted. Participants were recruited from $\mathrm{CHCs}$ run by three research centers (The Rehabilitation Hospital affiliated to Fujian University of Traditional Chinese Medicine, Fuzhou, China; The First Affiliated Hospital of Henan University of TCM Zhengzhou, China; and The Second Affiliated Hospital of Shandong University of Traditional Chinese Medicine, Jinan, China) via newspaper publicity, posting up posters, sending leaflets and referrals from neurologists and physical therapists. Ethical approvals were granted by ethics committees in all research centers. All participants were fully informed about the protocol and signed the written informed consent form prior to participation.

Inclusion criteria included the following: (1) Aged 45 to 75 years; (2) Diagnosed with stroke according to the criteria adopted by the Fourth National Cerebral Vascular Disease Conference [31] and confirmed by CT or MRI; (3) First onset of stroke more than 3 months prior; (4) Balance dysfunctions caused by stroke rather than other encephalopathies; (5) Ability to walk more than $6 \mathrm{~m}$ independently or assisted; (6) Agreement from patient or his/ 
her legal guardian and signed written informed consent; and (7) Mini-Mental State Exam score $>24$ and ability to understand, receive guidance and implement the exercises. The exclusion criteria included the following: (1) Existing diseases affecting training; (2) Impaired vestibular function; (3) Severe visual or hearing impairments that affected the training; (4) Sensory aphasia; (5) Prior Tai Chi Chuan experience in the last 6 months; (6) Serious complications after stroke; (7) Serious medical conditions such as severe heart disease, cancer, or gastrointestinal hemorrhage; and (8) Participation in other clinical trials that affected the results of this study.

\section{Sample size}

The sample size for the study was estimated based on our preliminary experiment, which suggested an intervention group would have a mean increase of 8.5 points $(\mathrm{SD}=2.8)$ on the BBS. We expected that 25 eligible cases would be enrolled in each cluster $(\mathrm{m}=25)$ based on the results of a pre-epidemiological investigation. With a type I error of 5 and $90 \%$ power, along with a 0.05 correlation coefficient intra- $\mathrm{CHC}$, a sample size of 114 was required according to the formula [32]:

$$
n=\frac{2\left[\left(\mu_{\alpha}+\mu_{\beta}\right)^{2} \sigma^{2}\right]}{\sigma^{2}}[1+(m-1) \rho]
$$

To allow for a $10 \%$ dropout rate, 125 participants were required for each group.

\section{Randomization and blinding}

To prevent cross-contamination, residents of each $\mathrm{CHC}$ were defined as a cluster. CHCs were randomly assigned to either the TC group or the control group in a 1:1 ratio via the PLAN algorithm in the statistical software SAS version 8.2 (SAS Institute, Inc., Cary, NC). The CHCs randomization program was safe-guarded by a specified project manager who did not participate in any other processes. Although it was impossible to blind the exercise coaches and subjects in this study, the coaches did not participant in any assessment of outcomes. The screeners, outcome evaluators and statisticians were blind throughout the study. Meanwhile, all outcome assessors and the data analyst did not participate in screening or assigning.

\section{Intervention}

The participants were allowed to maintain routine medical therapy (such as the management of the blood pressure, blood glucose, and accompanying symptoms et al.) depending on personal situation and visit their primary care physicians during the study period. Both groups received health education via a bulletin board, pamphlets and lectures. Both programs were carried out in the CHCs. All subjects completed a 12-week intervention.

Tai Chi Yunshou exercise occurred five times per week for $60 \mathrm{~min}$ each session. The form originated from the 24 short-form Tai Chi Chuan exercise normed by General Administration of Sports of China [33]. To exercise wave hands in the cloud, one must stand straight, move arms and legs with the waist at the axis, and breathe in a relaxed manner. The details of Tai Chi Yunshou exercise was reported at protocol of this study [34]. Each session comprised $45 \mathrm{~min}$ of exercise plus a 15-min warm-up and cool-down. Exercise was directed by five qualified coaches with more than 5 years of experience in physical education. The coach can adjust the length of the training according to patients' personal situation, and allocate properly. Individuals who can not complete the exercise continuously could be allowed to finish intermittently. But each session should be no less than 15 min. Meanwhile, another supervisor who was trained and qualified by research team was responsible for recording the training of individuals to ensure the quality of training for each community.

Balance rehabilitation training was also carried out five times per week for $60 \mathrm{~min}$ each session. The training form originated from the 'Technical Specification of Common Rehabilitation Therapy' (2012) published by the Chinese Association of Rehabilitation Medicine [35]. The balance rehabilitation training includes static balance training, dynamic balance training, bobath training, walking training and so on according to the patient's functional level and condition.

\section{Follow-up period}

All subjects completed a 12 weeks of follow-up conducted by telephone weekly and home visits monthly. During follow-up period, no participants were asked to receive additional intervention.

\section{Outcome measures}

Demographic characteristics were obtained from an interviewer administered questionnaire at recruiting. All outcomes measures were assessed at baseline and at the end of week 4, week 8 , and week 12 of the intervention as well as at 6-week follow-up (week 18) and 12-week follow-up (week 24). The design of this study has been reported previously [34].

The Chinese version of Berg Balance Scale (BBS), with good reliability and validity in Chinese stroke patients [36], was used to measure balance function as the primary outcome. The total of 14 items were used to measure static and dynamic aspects of balance function on a scale from 0 to 4 for each item [36]. Higher scores indicate better balance function. 
Static balance was measured by Single Leg Stance Test (SLST) [37]. Individuals were tested with eyes open; they were asked to stand on either their left or right leg and were instructed to keep their legs from touching and to maintain single-leg stance for as long as possible. The duration of standing was record. The best performance was recorded for each leg after three tests. A score was recorded as $0 \mathrm{~s}$ if the individual could not do it. Fugl-Meyer Assessment (FMA) was used to measure motor function, with 33 items that reflect upper-limb motor function and 17 items that reflect lower-limb motor function on a scale from 0 to 2 for each item [38]. Mobility was measured by Timed-Up-and-Go-Test (TUGT) [39]. The subjects were asked to rise from an armless chair, walk $3 \mathrm{~m}$ with orthosis or assistive device as required, turn round, and return to the seat as fast as possible. A sign was placed at the $3-\mathrm{m}$ point, and a research assistant blinded to the subject allocation recorded three times of a subject to complete the task via a stop-watch and calculated the average according to previous study $[28,37]$. ADL was measured by simplified Chinese version of Modified Barthel Index (MBI), with good reliability and validity in Chinese population, with the total 10 items [40]. Fear of falling was measured by Chinese version of Modified Falls Efficacy Scale (MFES), with good reliability and validity in Chinese population [41]. This scale asked participants to perceive their efficacy at avoiding falls during several relatively nonhazardous activities of daily living. The Medical Outcomes, Chinese version SF-36 including physical component summary (PCS) and mental component summary (MCS) parts, with good reliability and validity in Chinese population [42], was used to measure the quality of life, with higher scores predicting better perceived quality of life. Depression was measured by Beck Depression Inventory (BDI), a self-rating scale widely used in the assessment of depressive symptoms [43]. The BDI contains 21 groups of statements that describe mental conditions that are scored from 0 to 3 for each item. Higher scores indicate more severe depression [43].

\section{Safety evaluation}

Any adverse events that occurred were recorded on a case report form. The adverse events were defined as any unfortunate medical events incident during the study period (such as cardiovascular events, cerebral vascular events and falls).

\section{Statistical analysis}

All statistical analyses were performed using IBM SPSS for Windows (version 20.0). A two-sided $P$ value $\leq 0.05$ was considered to be statistically significant. The outcomes were analyzed on an intention-to-treat (ITT) basis. The missing data were imputed by using the Fully
Conditional Specification (FCS) algorithm of multiple imputations, which is an iterative Markov Chain Monte Carlo (MCMC) method (random number: 20171030). All continuous variables are described using means and standard deviations or median and inter-quartile range. T-tests or Mann-Whitney tests were used for continuous variables and Pearson's chi-squared or Fisher's exact tests were used for categorical variables to compare differences between groups. The repeated measures analysis of varianc was used to measured between-group differences. The variables of FMA, MBI, MEFS, BDI, which was different between groups at baseline, were treated as a covariate. The level of significance was 0.05 being the Bonferroni-adjusted alpha for across-time comparisons within the groups.

\section{Results}

Participant flow was showed as Fig. 1. Five participants in the TC group and one in balance rehabilitation group were dropped out before baseline assessments Therefore, they were not included in the ITT analysis. During the entire study period, eight participants in the TC group dropped out and eleven in the control group dropped out, and 112 (89.6\%) participants in the TC group and $113(90.4 \%)$ in the control group completed the study. The Fisher's exact text indicated that there was no significant difference in the dropout rates between groups $\left(\chi^{2}=0.413, P=0.521\right.$, Table 1$)$. However, not all individuals adhered to the complete training plan. The reasons given for absence including visits to physicians, health examinations, family dinners or other commitments that conflicted with training time. Finally, of the total 112 participants in the TC group, most participants had an attendance rate greater than $75 \%$ (48 participants attended more than $85 \%$ of the time and 59 participants, $76-85 \%)$, and a few subjects had an attendance rate of $75 \%$ or less (5 participants).

A total of 244 participants were included in final analysis; $74.6 \%$ were male, the average age was $60.9 \mathrm{y}$ in the TC group and $60.1 \mathrm{y}$ in the control group, and $71.7 \%$ had an ischemic stroke. The average length of the disease course was 14.5 months in the TC group and 14.3 months in the control group. There were no significant differences in baseline demographic characteristics between groups (including sex, age, education, duration of disease, MMSE, BBS, SLST, SF-36), and there were significant differences in FMA, MEFS, TUGT, MBI, and BDI $(P<0.05)$ between groups (Table 2).

Both Tai Chi Yunshou exercise and balance rehabilitation training had showed improvement by end of 12 weeks intervention over times (Fig. 2). The changes in outcomes for the Tai Chi Yunshou and balance rehabilitation group from baseline to the end of the 12 weeks intervention and to the end of the 12 weeks follow-up 


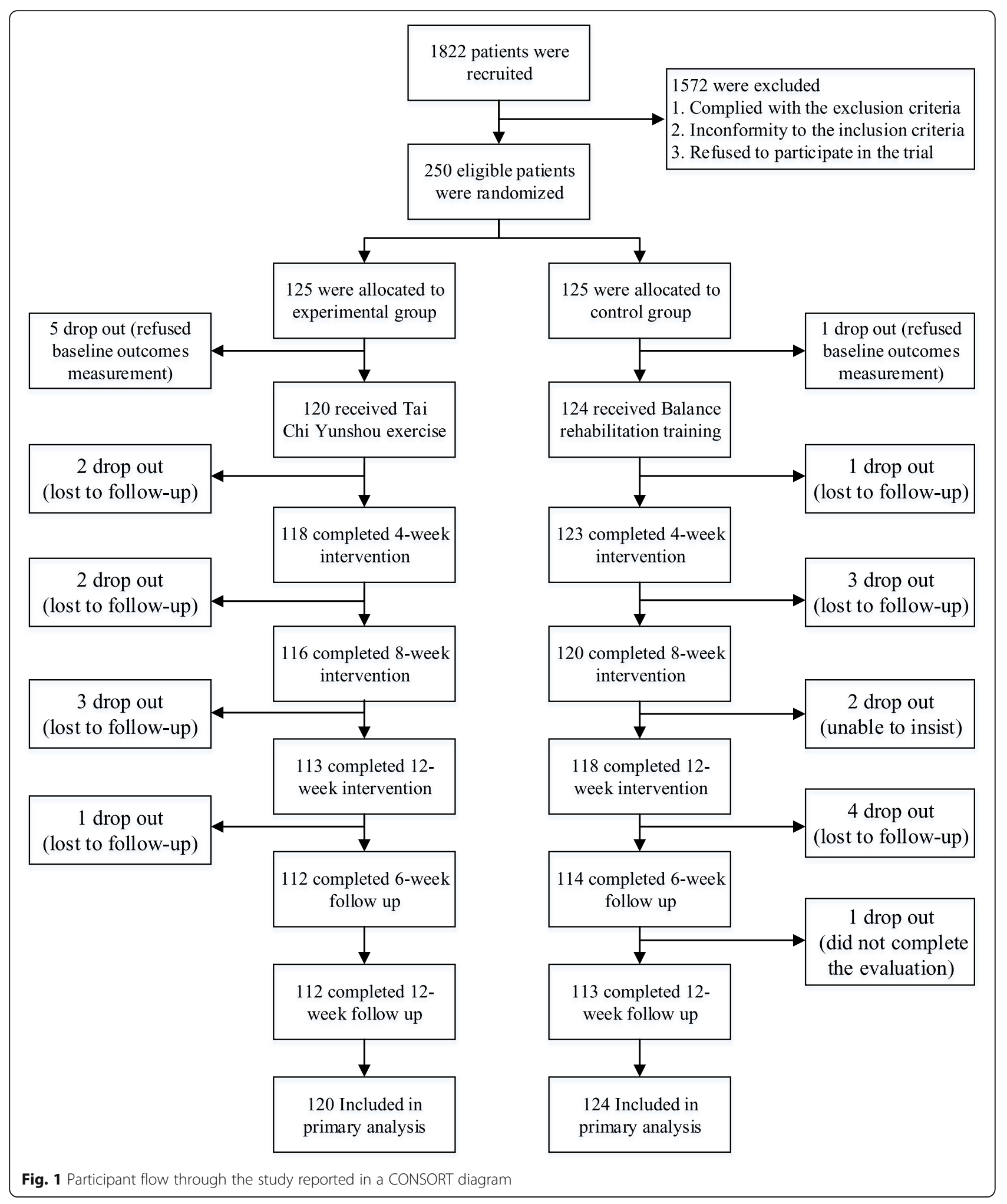

Table 1 Comparison of missing cases between two groups

\begin{tabular}{lllll}
\hline & $\begin{array}{l}\text { Tai Chi } \\
(n=120)\end{array}$ & $\begin{array}{l}\text { Control } \\
(n=124)\end{array}$ & $x^{2}$ value & $P$ value \\
\hline End of research (missing data/ complete data) $(n)$ & $8 / 112$ & $11 / 113$ & 0.413 \\
\hline
\end{tabular}


Table 2 Baseline demographic characteristics for the 2 groups

\begin{tabular}{|c|c|c|c|c|}
\hline \multirow[t]{2}{*}{ Parameters } & \multirow{2}{*}{$\begin{array}{l}\text { Tai Chi } \\
(n=120)\end{array}$} & \multirow{2}{*}{$\begin{array}{l}\text { Control } \\
(n=124)\end{array}$} & \multicolumn{2}{|l|}{ Group Difference } \\
\hline & & & $\overline{T\left(Z / F, X^{2}\right) \text { value }}$ & $P$ value \\
\hline Age, mean \pm SD (years) & $60.9 \pm 8.7$ & $60.1 \pm 8.6$ & $T=0.782$ & $0.435^{b}$ \\
\hline Sex (male, \%) & $83(69.2 \%)$ & 99 (79.8\%) & $x^{2}=3.665$ & $0.056^{\mathrm{a}}$ \\
\hline Education (years) & $9.6 \pm 3.9$ & $9.6 \pm 3.8$ & $Z=-0.226$ & $0.821^{c}$ \\
\hline Duration of disease (month) & $14.5 \pm 18.1$ & $14.3 \pm 22.1$ & $Z=-0.046$ & $0.963^{c}$ \\
\hline Type of stroke (Ischemic, \%) & $89(74.2 \%)$ & $86(69.4 \%)$ & $x^{2}=0.696$ & $0.404^{\mathrm{a}}$ \\
\hline Hemiplegia side & & & $x^{2}=5.124$ & $0.163^{\mathrm{a}}$ \\
\hline Left, \% & 67 (55.9\%) & $64(51.6 \%)$ & & \\
\hline Right, $\%$ & $36(30.0 \%)$ & $51(41.1 \%)$ & & \\
\hline Bilateral,\% & $3(2.5 \%)$ & $2(1.6 \%)$ & & \\
\hline No hemiplegia,\% & $14(11.6 \%)$ & $7(5.7 \%)$ & & \\
\hline Handedness ( $L, \%)$ & 119 (99.2\%) & $122(98.4 \%)$ & $x^{2}=0.305$ & $0.581^{\mathrm{a}}$ \\
\hline MMSE(score range: 0-30) & $27.2 \pm 1.6$ & $27.3 \pm 1.6$ & $Z=-0.599$ & $0.549^{c}$ \\
\hline BBS(score range: 0-56) & $33.5 \pm 13.7$ & $32.81 \pm 12.0$ & $Z=-0.686$ & $0.493^{c}$ \\
\hline \multicolumn{5}{|l|}{ SLST (s) } \\
\hline healthy side & $12.7 \pm 20.5$ & $8.2 \pm 12.7$ & $Z=-1.566$ & $0.117^{c}$ \\
\hline affected side & $3.0 \pm 6.1$ & $2.6 \pm 4.8$ & $Z=-0.878$ & $0.380^{c}$ \\
\hline FMA(score range: 0-100) & $53.6 \pm 25.3$ & $47.0 \pm 24.0$ & $T=2.082$ & $0.038^{b}$ \\
\hline TUGT (s) & $55.1 \pm 73.9$ & $42.0 \pm 28.0$ & $Z=-1.576$ & $0.115^{c}$ \\
\hline MBI(score range: 0-100) & $76.0 \pm 20.7$ & $71.0 \pm 19.1$ & $Z=-2.320$ & $0.020^{c}$ \\
\hline MEFS(score rang:1-140) & $77.5 \pm 35.6$ & $58.7 \pm 34.8$ & $Z=4.185$ & $<0.001^{\mathrm{c}}$ \\
\hline \multicolumn{5}{|l|}{ SF-36 } \\
\hline PCS(score range: 0-400) & $158.4 \pm 77.3$ & $152.0 \pm 80.6$ & $Z=-0.716$ & $0.474^{c}$ \\
\hline MCS(score range: 0-400) & $167.3 \pm 91.3$ & $177.9 \pm 77.0$ & $T=-0.977$ & $0.330^{b}$ \\
\hline BDI(score range:0-63) & $18.3 \pm 11.8$ & $14.4 \pm 10.2$ & $T=2.847$ & $0.005^{b}$ \\
\hline
\end{tabular}

All continuous variables are described using means and standard deviations

${ }^{a}$ performaned by Pearson $X^{2}$ test; ${ }^{b}$ performaned by the t-test; ${ }^{c}$ performaned by Mann-Whitney test

Abbreviations: MMSE the Mini-Mental State Examination, BBS Berg Balance Scale, SLST Single leg stance test, TUGT Timed-Up-and-Go test, FMA Fugl-Meyer, MBI

Modified Barthel Index, MFES Modified Falls Efficacy Scale, SF-36 the Mos Study 36-Item Short-Form Health Survey, PCS physical component summary, MCS mental component summary, $B D I$ Beck Depression Inventory

was showed in Table 3. For the primary outcome, BBS, the similar impovement was observed between groups on the change of week 12 to baseline and change of week 24 to baseline $(P=0.915, P=0.715$, respectively), and representing no significant difference after adjustment for baseline using a general linear model $(P=$ 0.531, Table 4). For another balance parameter, SLST, the similar improvement was also observed between Tai Chi Yunshou and balance rehabilitation training after adjustment for baseline using a general linear model $(P$ $=0.102, P=0.221$, respectively, Table 4$)$. Of the parameter for quality of life, the similar effect of Tai Chi Yunshou and balance rehabilitation training was observed after adjustment for baseline using a general linear model (PCS: $P=0.063$ and MCS: $P=0.052$, respectively, Table 4).

Futhermore, the Tai Chi Yunshou had more positive effects on the motor function, fear of falling and depression than balance rehabilitation training. For the parameter of motor function, the difference between two groups in the FMA was observed after adjustment for baseline using a general linear model $(P=0.022)$. The difference between two groups was also observed on fear of falling after adjustment for baseline using a general linear model $(P<0.001)$. The significant difference between groups was observed on depression parameter $(P=0.035)$.

\section{Safety}

There was no adverse events related to Tai Chi Yunshou occurred in this trial.

\section{Discussion}

In this cluster randomized controlled trial, we defined the balance rehabilitaion training as positive control, which is known as standard method for the balance rehabilitation of the stroke surivors according the 

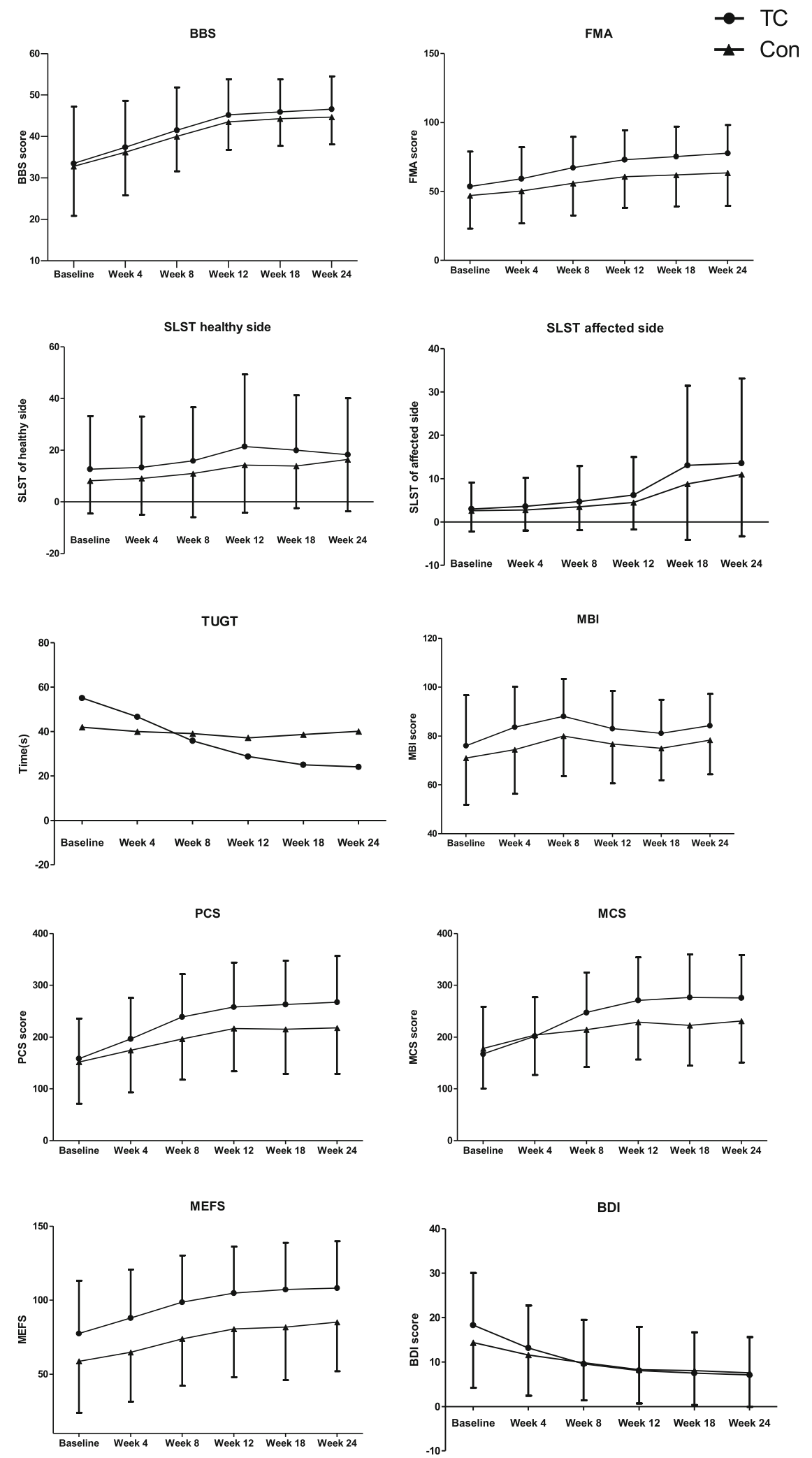

Fig. 2 Comparison between Tai Chi Yunshou and control ggroups in the outcomes over time 


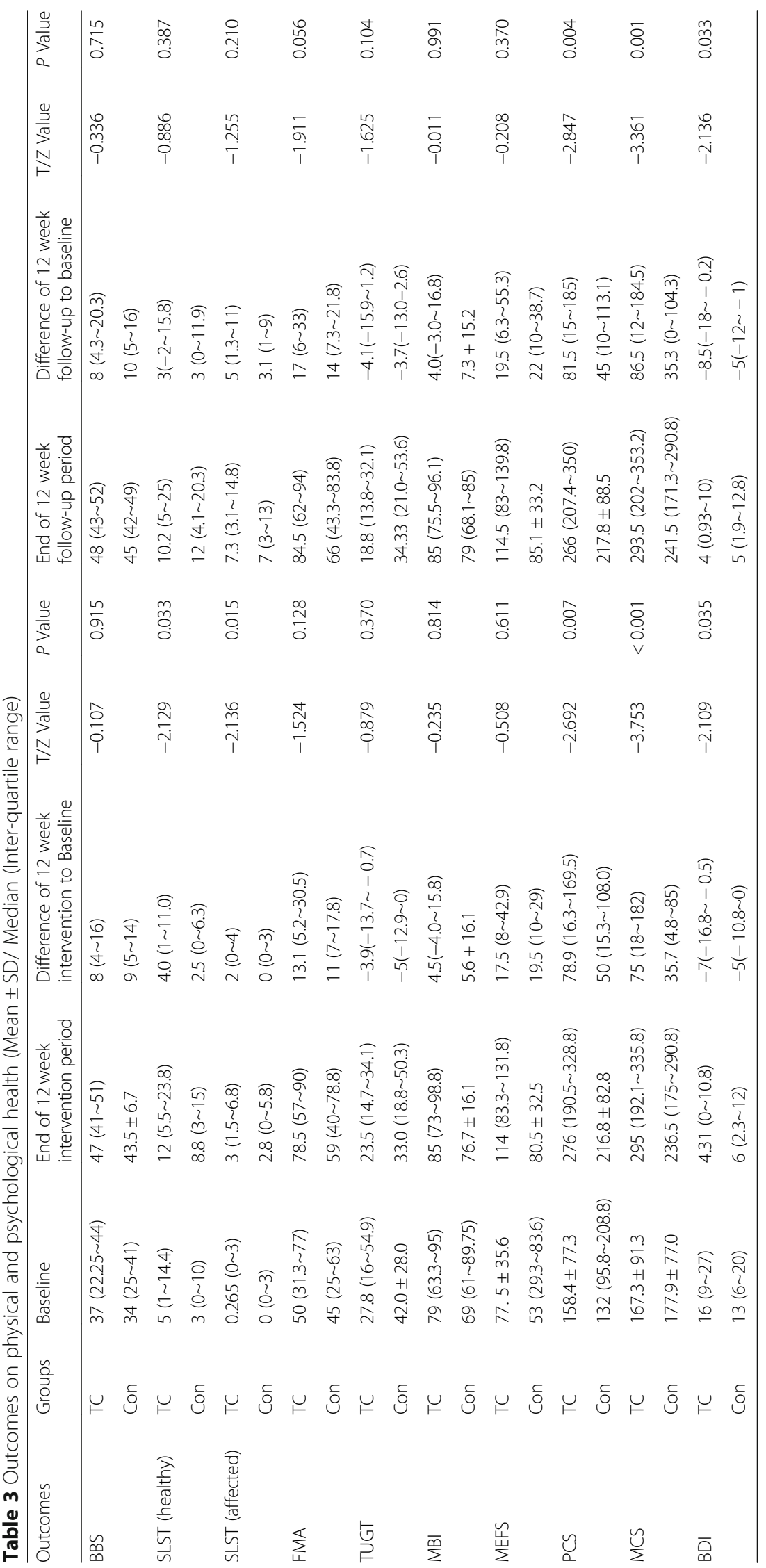


Table 4 The effects of the Tai Chi Yunshou on the outcomes

\begin{tabular}{|c|c|c|c|c|c|}
\hline Outcomes (score range) & Scourses & Sum of Squares & F Value & $P$ Value & effect size Value \\
\hline \multirow[t]{3}{*}{$\overline{B B S}(0-56)$} & Time & 4060.833 & 38.407 & $<0.001$ & 0.139 \\
\hline & Group & 63.321 & 0.394 & 0.531 & 0.002 \\
\hline & GroupxTime & 120.37 & 1.138 & 0.325 & 0.005 \\
\hline \multirow[t]{3}{*}{ SLST(s) (healthy side) } & Time & 8146.268 & 12.521 & $<0.001$ & 0.050 \\
\hline & Group & 670.850 & 2.689 & 0.102 & 0.011 \\
\hline & GroupxTime & 1103.528 & 11.696 & 0.168 & 0.007 \\
\hline \multirow[t]{3}{*}{ SLST(s) (affected side) } & Time & 219.337 & 0.641 & 0.486 & 0.003 \\
\hline & Group & 79.723 & 1.507 & 0.221 & 0.006 \\
\hline & GroupxTime & 193.981 & 0.567 & 0.521 & 0.002 \\
\hline \multirow[t]{3}{*}{ FMA(0-100) } & Time & $14,548.498$ & 41.520 & $<0.001$ & 0.148 \\
\hline & Group & 9796.797 & 5.332 & 0.022 & 0.022 \\
\hline & GroupxTime & 2613.783 & 7.460 & $<0.001$ & 0.030 \\
\hline \multirow[t]{3}{*}{ TUGT(s) } & Time & 746.657 & 0.330 & 0.645 & 0.001 \\
\hline & Group & 22.076 & 0.042 & 0.839 & $<0.001$ \\
\hline & GroupxTime & $21,802.737$ & 9.627 & 0.001 & 0.020 \\
\hline \multirow[t]{3}{*}{ MBI(0-100) } & Time & 4212.047 & 15.487 & $<0.001$ & 0.061 \\
\hline & Group & 963.320 & 1.511 & 0.220 & 0.006 \\
\hline & GroupxTime & 629.350 & 2.314 & 0.080 & 0.010 \\
\hline \multirow[t]{3}{*}{ MEFS(1-140) } & Time & $21,175.317$ & 21.490 & $<0.001$ & 0.082 \\
\hline & Group & $14,144.527$ & 30.760 & $<0.001$ & 0.114 \\
\hline & GroupxTime & 1662.543 & 632.574 & 0.175 & 0.007 \\
\hline \multicolumn{6}{|l|}{ SF-36 } \\
\hline \multirow[t]{3}{*}{ PCS $(0-400)$} & Time & $69,737.418$ & 7.667 & $<0.001$ & 0.031 \\
\hline & Group & $11,316.527$ & 3.501 & 0.063 & 0.014 \\
\hline & GroupxTime & $42,651.626$ & 4.689 & 0.005 & 0.019 \\
\hline \multirow[t]{3}{*}{$\operatorname{MCS}(0-400)$} & Time & $19,770.236$ & 1.899 & 0.136 & 0.008 \\
\hline & Group & $10,608.506$ & 3.825 & 0.052 & 0.016 \\
\hline & GroupxTime & $94,181.540$ & 9.047 & $<0.001$ & 0.037 \\
\hline \multirow[t]{3}{*}{ BDI(0-63) } & Time & 4374.532 & 37.971 & $<0.001$ & 0.137 \\
\hline & Group & 1386.080 & 4.490 & 0.035 & 0.018 \\
\hline & GroupxTime & 1246.265 & 10.818 & $<0.001$ & 0.043 \\
\hline
\end{tabular}

Abbreviations: Berg Balance Scale BBS, Fugl-Meyer Assessment FMA, Single Leg Stance Test SLST, Timed-Up-and-Go-Test TUGT, Modified Barthel Index MBI, Modified Falls Efficacy Scale MFES, the Mos Study 36-Item Short-Form Health Survey SF-36, physical component summary PCS, mental component summary MCS, Beck Depression Inventory BDI

guideline [44]. Compared Tai Chi Yunshou exercise with positive control on the physical and mental health of stroke survivors, we found the similar effectiveness of two intervention methods for parameters of balance ability (BBS, SLST and TUGT), activities of daily living (MBI), quality of life (PCS and MCS) over a 12-week intervention period. Furthermore, the improvement of motor function (FMA score), fear of falling (MFES), depression (BDI) in the Tai Chi Yunshou exercise is more effective than balance rehabilitation over a 12-week intervention period, which indicated that Tai Chi Yunshou has benefits on balance function, motor function, fear of falling and quality of life for community-based stroke survivors. The was no adverse events reported during the intervention period, which suggested that Tai Chi Yunshou exercise is safe for this population.

Tai Chi Chuan is a traditional Chinese mind-body exercise. Participants in Tai Chi Chuan were asked to shift their center of gravity slowly and at a constant speed between the two legs, revolving around the waist, while at the same time keeping the body upright, the shoulders and head in an upright posture and relaxed, and the spine comfortably aligned. Therefore, this type exercise program is expected to improve proprioceptive 
awareness and kinesthetic sense [45-47]. Previous studies had demonstrated that Tai Chi Chuan exercise has benefits on balance ability and gait speed for stroke survivors $[16,18,48]$. The improvement of Tai Chi Chuan on psychological symptoms, such as depression has also been reported $[49,50]$. However, the Tai Chi Chuan used in previous study were 6-form [27], 12-form [17] or 24-form [16] style which make the exercise challenging for the stroke survivors. The Tai Chi Yunshou (wave hands in the cloud) movement is the basic and "mother" form of all styles of Tai Chi Chuan. During Tai Chi Yunshou exercise, head-and-eye movements, the trunk and limbs movement are synchronous and frequent changes of head-and-body orientation are required. This may result in a better combination of the visual and vestibular systems for balance control. To this end, the single form (waves hands in cloud movement) of Tai Chi Chuan was used in this study to improvement the physical and mental health for stroke survivors.

In this study, balance ability was evaluated using BBS, SLST. The improvement of the balance ability between two group was not showed statistical significance. This is in agreement with previous research [26]. Hart et al. reported that both Tai Chi Chuan and general physical therapy can improve the balance ability (BBS, standing on one leg) and speed of walking and there was no significant difference between groups. The authors also suggested that Tai Chi Chuan can be used as an alternative and complementary therapy for the stroke with balance impairment [26]. In contrast, $\mathrm{Au}$-Yeung indicated that a significant improvement in TUGT of stroke patients performed Tai Chi Chuan once a week for 12-week compared to control group [17]. In addition, Taylor-Piliae and Coull reported that stroke survivors exercised Tai Chi Chuan had balance and walking speed improved [16]. The explain maybe that those studies defined usual care as intervention of control group which can not improve the balance ability and mobility. For the quality of life parameter, both Tai Chi Yunshou exercise and balance rehabilitaion can improve the quality of life for the stroke survivors. This is consistent with previous research $[15,18]$, which demonstrated that 12-week Tai Chi practice could benefit the quality of life of stroke survivors. The reason for this finding may be that this group activity provides social support and participation, which improves or preserves the quality of life.

Furthermore, we demonstrated that the Tai Chi Yunshou exercise and balance rehabilitation training can effective in improving the motor function, fear of falling and depression. A previous study with post-stroke survivors enrolled demonstrated an improvement in motor function after a 4-week Tai Chi exercise program compared to rehabilitation training [51], which is in accordance with current study. As a moderate intensity aerobic exercise combining static and dynamic movements [30], Tai Chi Yunshou asks subjects to place the center of their body weight on two legs, which can enhance the muscular strength of the lower limbs [34]. This may be the reason why Tai Chi Yunshou improved the motor function of stroke survivors. Our result was also similar to a recent study conducted by Lin [52], who assessed the effectiveness of Tai Chi Chuan exercise on fall-related outcomes among frail adults compared with balance rehabilitation program. They found that Tai Chi Chuan was more effective in improving fear of falling than general physical therapy. The improvement in balance ability by Tai Chi Yunshou was not agree with changes in motor function as measured by FMA. This result elucidated that the effect of Tai Chi Yunshou exercise was specific to the task being trained, namely, balance ability by performing head, body with weight shifting. The Tai Chi Yunshou form do not stress turning. As a body-mind exercise, the meditative and relaxation training of Tai Chi has been shown to reduce anxiety and depression [53]. The reason for this finding may be that Tai Chi Yunshou emphasizes sustained attentional focus and mind-regulation during movement, which may impact cortisol and other stress-related pathways.

More than $85 \%$ of the subjects completed the experiment, and at least $95 \%$ of the subjects were included in the analysis. Only 20 cases fell off (a drop-out rate of $8 \%)$. No adverse events occurred. A series of measures were carried out to ensure the adherence and safety of the participants. First, our class sizes were small (maximum of five/group) so that the intervener could provide on-site guidance and supervision sufficiently. Second, $\mathrm{CHCs}$ near the subjects were selected as training venues to ensure compliance. Third, those who could not exercise for $1 \mathrm{~h}$ continuously could complete the session in sections, which were not less than $15 \mathrm{~min}$ each section. Fourth, subjects with severe upper limb dysfunction were allowed to perform the exercises by using the unaffected hand to aid the affected hand. Additionally, health education was provided to all individuals throughout the intervention period. Weekly telephone follow-ups and monthly family follow-ups were used to improve the return ratio. Another important reason we hypothesize for the small dropout rate is that Tai Chi Yunshou exercise is constructed as a group training, community-based program, in which individuals can receive encouragement including social support and knowledge acquisition [2].

\section{Conclusion}

In conclusion, the present study demonstrated that both a 12-week Tai Chi Yunshou exercise and balance 
rehabilitation led to impoved balance ability and functional mobility, and both are suitable community-based programs that may benefit for stroke recovery and community reintegration. Our data suggested that a 12-week Tai Chi Yunshou intervention was more effective in improving motor function, fear of falling and quality of life than balance rehabilitation. The basic form of Tai Chi Chuan, waves hands in cloud movement, can be used as an alternative and complementary therapy for the community-based stroke survivors with balance impairment.

\section{Limitations}

There are also some limitations in this study. First, although it was impossible to blind the participants and instructors in this study, the outcome evaluators and analysts were blinded to reduce bias. Second, participants were not asked to record their daily exercise, including the type, exercise time, frequency, etc., during the study, which could also be a confounding factor that affected the results. Additionally, there was no supervision during the follow-up period, which may have magnified the effects due to the self-initiated exercise of patients. In addition, the observed improvements in physical function among participants in this study may have occurred as a result of a learning effect, associated with repeated use of the study measures to assess changes after the 12-week intervention. We did not check the intensity of two interventions, which can effect the results.

\section{Abbreviations \\ ADL: Activities of daily living; BBS: Berg Balance Scale; BDI: Beck Depression Inventory; CHCs: Community health centers; FMA: Fugl-Meyer Assessment; ITT: Intention-to-treat; MBI: Modified Barthel Index; MFES: Modified Falls Efficacy Scale; SF-36: The Medical Outcomes Study 36-Item Short-Form Health Survey; SLST: Single Leg Stance Test; TUGT: Timed-Up-and-Go-Test \\ Acknowledgments \\ This study is funded by the Special Scientific Research Fund of Public Welfare Profession of China (Grant No. 201307004), Ministry of Science and Technology and Ministry of Finance of the People's Republic of China. It is supported by National-Local Joint Engineering Research Center of Rehabilitation Medicine Technology and National Traditional Chinese Medicine Rehabilitation Research Center of State Administration of Traditional Chinese Medicine of the People's Republic of China.}

\section{Availability of data and materials}

The datasets used and/or analysed during the current study are available from the corresponding author on reasonable request.

\section{Informed consent}

Written informed consent was obtained from all individual participants included in the study.

\section{Authors' contributions}

JT and LDC conceived and designed the research; GLX and TR drafted the article; LLL, ZKL, and JSW acquired data; TSX, MGY, and JMFan are research assistants who will help organize to actively recruit, train, assess outcome, data manage; SFL, XDF, and LL supervised the research programme; YX interpreted data results; JT and LDC revised the manuscript; All authors meet the criteria for authorship stated in the Uniform Requirements for Manuscripts Submitted to Biomedical Journals. All authors read and approved the manuscript.

\section{Ethics approval and consent to participate}

All procedures performed in studies involving human participants were in accordance with the ethical standards of the institutional and/or national research committee and with the 1964 Helsinki declaration and its later amendments or comparable ethical standards. Ethical approvals have already been granted by ethics committees in all research centers, including the ethics committee of The Rehabilitation Hospital affiliated to Fujian University of Traditional Chinese Medicine (2013KY-006-01, approval received in July 2013), the ethics committee of The First Affiliated Hospital of Henan University of TCM (2014HL010, approval received in March 2014) and the ethics committee of The Second Affiliated Hospital of Shandong University of Traditional Chinese Medicine (2013KY-006-01, approval received in March 2014).

\section{Consent for publication}

Not applicable.

\section{Competing interests}

All authors declare that they have no competing of interest.

\section{Publisher's Note}

Springer Nature remains neutral with regard to jurisdictional claims in published maps and institutional affiliations.

\section{Author details}

${ }^{1}$ College of Rehabilitation Medicine, Fujian University of Traditional Chinese Medicine, 1 Huatuo Road, Minhou Shangjie, Fuzhou 350122, Fujian, China. ${ }^{2}$ Affiliated Rehabilitation Hospital, Fujian University of Traditional Chinese Medicine, Fuzhou, China. ${ }^{3}$ Traditional Chinese Medicine Rehabilitation Research Center of State Administration of Traditional Chinese Medicine, Fuzhou, People's Republic of China. ${ }^{4}$ Rehabilitation medical technology Joint National Local Engineering Research Center, Fuzhou, China. ${ }^{5}$ Fujian Collaborative Innovation Center for Rehabilitation Technology, Fuzhou, China. ${ }^{6}$ The First Affiliated Hospital of Henan University of Traditional Chinese Medicine, Zhengzhou, China. ${ }^{7}$ The Second Affiliated Hospital of Shandong University of Traditional Chinese Medicine, Jinan, China.

Received: 13 April 2018 Accepted: 28 November 2018 Published online: 12 December 2018

\section{References}

1. Feigin $\mathrm{VL}$, Forouzanfar MH, Krishnamurthi R, Mensah GA, Connor M, Bennett DA, Moran AE, Sacco RL, Anderson L, Truelsen T, O'donnell M, Venketasubramanian N, Barker-Collo S, Lawes CMM, Wang W, Shinohara Y, Witt E, Ezzati M, Naghavi M, Murray C, Group R F S G a T G S E. Global and regional burden of stroke during 1990-2010: findings from the global burden of disease study 2010. Lancet. 2014;383: 245-54.

2. Reed M, Harrington R, Duggan A, Wood VA. Meeting stroke survivors' perceived needs: a qualitative study of a community-based exercise and education scheme. Clin Rehabil. 2010;24:16.

3. Geurts AC, De Haart M, Van Nes IJ, Duysens J. A review of standing balance recovery from stroke. Gait Posture. 2005;22:267-81.

4. Tyson SF, Hanley M, Chillala J, Selley A, Tallis RC. Balance disability after stroke. Phys Ther. 2006:86:30-8.

5. Oliveira CB, Medeiros RT, Greters MG, Frota N a F, Lucato LT, Scaff M, Conforto $A B$. Abnormal sensory integration affects balance control in hemiparetic patients within the first year after stroke. Clinics. 2011;66: 2043-8.

6. Michael K, Goldberg AP, Treuth MS, Beans J, Normandt P, Macko RF. Progressive adaptive physical activity in stroke improves balance, gait, and fitness: preliminary results. Top Stroke Rehabil. 2009;16:133-9.

7. Michael KM, Allen JK, Macko RF. Reduced ambulatory activity after stroke: the role of balance, gait, and cardiovascular fitness. Archives of Physical Medicine \& Rehabilitation. 2005;86:1552-6.

8. Allison R, Dennett R. Pilot randomized controlled trial to assess the impact of additional supported standing practice on functional ability post stroke. Clin Rehabil. 2007;21:614

9. Chan DY, Chan CC, Au DK. Motor relearning programme for stroke patients: a randomized controlled trial. Clin Rehabil. 2006;20:191.

10. Williams TM D L P T B a S L S. Barriers and facilitators to exercise among stroke survivors. Rehabilitation nursing. 2007;32:253-60. 
11. Lubetzky-Vilnai A, Kartin D. The effect of balance training on balance performance in individuals poststroke: a systematic review. Journal of Neurologic Physical Therapy Jnpt. 2010;34:127.

12. Benjamin EJ, Blaha MJ, Chiuve SE, Cushman M, Das SR, Deo R, De SF, Floyd J, Fornage M, Gillespie C. Heart disease and stroke statistics - 2017Update: a report from the American Heart Association. Circulation. 2017;131:e29.

13. Mead $G$, Bernhardt J. Physical fitness training after stroke, time to implement what we know: more research is needed. Int I Stroke. 2011;6:506-8.

14. Wooton AC. An integrative review of tai chi research: an alternative form of physical activity to improve balance and prevent falls in older adults. Orthop Nurs. 2010:29:108.

15. Ho TJ, Liang WM, Lien CH, Ma TC, Kuo HW, Chu BC, Chang HW, Lai JS, Lin JG. Health-related quality of life in the elderly practicing T'ai chi Chuan. J Altern Complement Med. 2007;13:1077-83.

16. Taylor-Piliae RE, Coull BM. Community-based Yang-style tai chi is safe and feasible in chronic stroke: a pilot study. Clin Rehabil. 2012;26:121.

17. Au-Yeung SS, Hui-Chan CW, Tang JC. Short-form tai chi improves standing balance of people with chronic stroke. Neurorehabil Neural Repair. 2009;23: 515-22.

18. Taylor-Piliae RE, Hoke TM, Hepworth JT, Latt LD, Najafi B, Coull BM. Effect of tai chi on physical function, fall rates and quality of life among older stroke survivors. Arch Phys Med Rehabil. 2014;95:816-24.

19. Azimzadeh E, Hosseini MA, Nourozi K, Davidson PM. Effect of tai chi Chuan on balance in women with multiple sclerosis. Complement Ther Clin Pract. 2015;21:57-60

20. Li F, Harmer P, Fitzgerald K, Eckstrom E, Stock R, Galver J, Maddalozzo G, Batya SS. Tai chi and postural stability in patients with Parkinson's disease. N Engl J Med. 2012;366:511-9.

21. Harkin EA, Lewis KA, Burton KA. Exploring tai chi as an early intervention to improve balance and reduce falls among stroke survivors - towards a feasibility study. European Journal of Integrative Medicine. 2016;8:591.

22. Gatts SK, Woollacott MH. How tai chi improves balance: biomechanics of recovery to a walking slip in impaired seniors. Gait \& Posture. 2007;25:205-14.

23. Zhang L, Layne C, Lowder T, Liu J. A review focused on the psychological effectiveness of tai chi on different populations. Evid Based Complement Alternat Med. 2012;2012:678107.

24. Weimo Z, Shangyi G, Yubing Y. Clinical implications of tai chi interventions: a review. Am J Lifestyle Med. 2010:4:418-32.

25. Ding M. Tai chi for stroke rehabilitation: a focused review. Am. J. Phys. Med. Rehabil. 2012;91:1091.

26. Hart J, Kanner H, Gilboa-Mayo R, Haroeh-Peer O, Rozenthul-Sorokin N, Eldar R. Tai chi Chuan practice in community-dwelling persons after stroke. Int J Rehabil Res. 2004;27:303-4.

27. Zhang Y, Liu H, Zhou L, Chen K, Jin H, Zou Y, Li Z. Applying tai chi as a rehabilitation program for stroke patients in the recovery phase_ study protocol for a randomized controlled trial. Trials. 2014;15:184.

28. Kim H, Kim YL, Lee SM. Effects of therapeutic tai chi on balance, gait, and quality of life in chronic stroke patients. Int J Rehabil Res. 2015;38:156-61.

29. Simpson LA, Eng JJ, Tawashy AE. Exercise perceptions among people with stroke: barriers and facilitators to participation. Int J Ther Rehabil. 2011;18: 520-30

30. Taylor-Piliae RE, Haskell WL. Tai chi exercise and stroke rehabilitation. Top Stroke Rehabil. 2014;14:9-22.

31. Association C M. Various types of cerebrovascular disease diagnostic criteria. Zhonghua Shen Jing Ge Za Zhi. 1996:29:379.

32. Jing G, Shuigao J. Sample estimation: Cluster randomization test design. Chin J Health Stat. 2008;25:117-9.

33. Tong $Y$. Chinese national standard movements of tai chi competition 24 Chengdu: Chengdu erapress; 2009.

34. Tao J, Rao T, Lin L, Liu W, Wu Z, Zheng G, Su Y, Huang J, Lin Z, Wu J, Fang $Y$, Chen L. Evaluation of tai chi Yunshou exercises on community-based stroke patients with balance dysfunction: a study protocol of a cluster randomized controlled trial. BMC Complement Altern Med. 2015;15:31

35. Medicine $\mathrm{C}$ a O R, in Technical Specification of Common Rehabilitation Therapy 2013, People's Medical Publishing House: Beijing.

36. Dong-Mei J, Tie-Bin Y. Berg balance scale and its clinical application. Chin Rehabil Theory and Pract. 2002;8:155-7.

37. Kamide NSY, Shibata H. Effects on balance, falls, and bone mineral density of a home-based exercise program without home visits in communitydwelling elderly women: a randomized controlled trial. J Physiol Anthropol. 2009;28:115
38. Gladstone DJ, Danells CJ, Black SE. The Fugl-Meyer assessment of motor recovery after stroke: a critical review of its measurement properties. Neurorehabil Neural Repair. 2002;16:232

39. Barry E, Galvin R, Keogh C, Horgan F, Fahey T. Is the timed up and go test a useful predictor of risk of falls in community dwelling older adults: a systematic review and meta- analysis. BMC Geriatr. 2014;14:14

40. Yun M, Yuan-Yuan W, Tie-Bin Y. Validity and reliability of the simplified Chinese version of modified Barthel Index for Chinese stroke patients. Chin J Phys Med Rehabil. 2008;30:185-8.

41. Yan-Ping $H$, Xue-Qin $L$. Research of application of the modified fall efficacy scale of Chinese version in the aged in China. Chin J Nursing. 2007:42:19-21.

42. Biao Z, Kun C, Jun-Fang W, Yin-Yin W, Wei-Jun Z, Hui W. Reliability and validity of a Short-Form Health Survey Scale(SF-36),Chinese version in an elderly population of Zhejiang province in China. Chin J Epidemiol. 2008;29: 1193-8.

43. Mendelson M. An inventory for measuring depression. Arch Gen Psychiatry. 1961;4:561-71.

44. Winstein CJ, Stein J, Arena R, Bates B, Cherney LR, Cramer SC, Deruyter F, Eng JJ, Fisher B, Harvey RL. Guidelines for adult stroke rehabilitation and recovery: a guideline for healthcare professionals from the American Heart Association/American Stroke Association. Stroke. 2016;47:e98.

45. Zhang C, Sun W, Yu B, Song Q, Mao D. Effects of exercise on ankle proprioception in adult women during 16 weeks of training and eight weeks of detraining. Research in Sports Medicine. 2015:23:102-13.

46. Sun W, Zhang C, Song Q, Li W, Cong Y, Chang S, Mao D, Hong Y. Effect of 1-year regular tai chi on neuromuscular reaction in elderly women: a randomized controlled study. Research in Sports Medicine. 2016;24:145.

47. Sun W, Ma X, Wang L, Zhang C, Song Q, Gu H, Mao D. Effects of Tai Chi Chuan and brisk walking exercise on balance ability in elderly women: a randomized controlled trial. Mot Control. 2018;1:1-15.

48. Ti-Jun L, Ping Q, Xin-Zhi C. The effect of taijiquan to improve the balance function of stroke patients. Chin J Phys Med Rehabil. 2009;31:781-2.

49. Zheng G, Lan X, Li M, Ling K, Lin H, Chen L, Tao J, Li J, Zheng X, Chen B, Fang $Q$. Effectiveness of tai chi on physical and psychological health of college students: results of a randomized controlled trial. PLoS One. 2015;10: e0132605

50. Wang C, Bannuru R, Ramel J, Kupelnick B, Scott T, Schmid CH.Tai chi on psychological well-being: systematic review and meta-analysis. BMC Complement Altern Med. (2010-05- 21). 2010:10:23.

51. Zhou L, Li Z, Zhang Y, Chen K, Lin Y.Preliminary study on the effect of modified tai chi for motor function in patients with stroke. Chin J Integr Med Cardio-/Cerebrovasc Dis. 2015;13:878-80.

52. Lin MR, Hwang HF, Wang YW, Chang SH, Wolf SL. Community-based tai chi and its effect on injurious falls, balance, gait, and fear of falling in older people. Phys Ther. 2006;86:1189-201.

53. Wang C, Bannuru R, Ramel J, Kupelnick B, Scott T, Schmid CH. Tai chi on psychological well-being: systematic review and meta-analysis. BMC Complement Altern Med. 2010;10:1-16.

Ready to submit your research? Choose BMC and benefit from

- fast, convenient online submission

- thorough peer review by experienced researchers in your field

- rapid publication on acceptance

- support for research data, including large and complex data types

- gold Open Access which fosters wider collaboration and increased citations

- maximum visibility for your research: over $100 \mathrm{M}$ website views per year

At $\mathrm{BMC}$, research is always in progress.

Learn more biomedcentral.com/submission 